\title{
AAA: a rock and a hard place
}

\author{
Omesh Modgill, ${ }^{\star 1}$ Ginal Patel, ${ }^{2}$ Dapo Akintola, ${ }^{3}$ Olamide Obisesan ${ }^{4}$ and Harjit Tagar ${ }^{3}$
}

\section{Key points}

Ascertains the impact of the advice, analgesia and antibiotics (AAA) regimen upon prescribing patterns in primary and secondary dental care.
Discusses the challenges in performing appropriate antibiotic prescription during the COVID-19 pandemic and proposes approaches to remedy such challenges.
Discusses the rationale potentially underpinning antibiotic prescribing patterns during the COVID-19 pandemic and highlights the importance of further consideration of prudent antibiotic prescription in light of potential future mandatory closures of dental practices.

\begin{abstract}
Introduction This retrospective analysis sought to ascertain the effect of the advice, analgesia and antibiotics (AAA) regimen upon the appropriateness of antibiotic prescribing for those patients attending for emergency dental extraction at the Department of Oral Surgery, King's College Dental Hospital (KCDH), London. This has subsequently been used as a foundation upon which to discuss the potential factors that are likely to have had an effect upon the prescribing patterns of general dental practitioners (GDPs) throughout the United Kingdom (UK) at this time and possible future implications should the UK experience a second mandatory closure of primary care dental settings.
\end{abstract}

Materials and methods Retrospective data collection for patients attending for emergency dental extractions was performed at the Department of Oral Surgery, KCDH. Data were collected between March-June 2020 during KCDH's designation as an urgent dental care hub.

Results In total, 1,414 patients attended for emergency dental extraction. Four hundred and seventy-one (33.3\%) patients sought advice from their GDP before contacting KCDH's emergency dental triage service. Prior to attending KCDH for emergency dental extraction, 665 (47\%) patients were prescribed antibiotics by a primary care health provider.

Conclusion Our findings suggest that the AAA regimen may have inadvertently contributed to inappropriate prescription of systemic antibiotics by GDPs.

\section{Introduction}

Antimicrobial resistance (AMR) remains a global health challenge. Whilst antibiotic prescribing in primary care dental settings has likely reduced over the past decade, ${ }^{1}$ inappropriate antibiotic prescribing throughout dentistry persists. ${ }^{2}$ Systemic antibiotic prescriptions as a primary means for the management of inflammatory dental conditions in the absence of local and/or

'Specialist Oral Surgeon, King's College Dental Hospital, London, SE5 9RS, UK; ${ }^{2}$ Dental Core Trainee Year 1, King's

College Dental Hospital, London, SE5 9RS, UK; ${ }^{3}$ Consultant

Oral Surgeon, King's College Dental Hospital, London, SE5

9RS, UK; ${ }^{4}$ Consultant Oral Surgeon and Departmental Lead

King's College Dental Hospital, London, SE5 9RS, UK.

*Correspondence to: Omesh Modgill

Email address: omesh.modgill@nhs.net

Refereed Paper.

Accepted 26 November 2020

https://doi.org/10.1038/s41415-020-2594-3 systemic infection are ineffective in managing such symptoms, as these may unnecessarily persist. This approach therefore fails to act in patients' best interests and directly contravenes the prescribing dental practitioner's legal duty of care.

Globally, indiscriminate antibiotic prescribing contributes towards AMR and the emergence of several drug-resistant bacterial strains, ${ }^{3,4,5}$ thus further detrimentally impacting upon the ability of global healthcare systems to effectively manage conditions of bacterial aetiology.

Antibiotic prescribing guidance in the United Kingdom (UK) is provided by the Faculty of General Dental Practitioners (FGDP), ${ }^{6}$ Scottish Dental Clinical Effectiveness Programme (SDCEP) ${ }^{7}$ and the National Institute for Health and Care Excellence (NICE). ${ }^{8}$ Public Health England's (PHE's) 'Dental antimicrobial stewardship: toolkit' has collated much of this guidance and that produced by other organisations, such as the British Dental Association and British Association of Oral Surgeons, as a reference tool for dental practitioners when considering antibiotic prescription for patients.

Dental practices in the UK were mandated to temporarily cease routine dental activity from March $2020^{10}$ and permitted to resume services from 4 June 2020. During this timeframe, NHS England's guidance regarding the management of urgent dental conditions ${ }^{11}$ suggested the adoption and prescription of the 'triple A' regimen of advice, analgesia and antibiotics (AAA), as indicated. General dental practitioners (GDPs) were permitted to conduct telephone triage and assessment, and to manage patients accordingly. In cases where the AAA approach was considered ineffective, GDPs were advised to refer to locally established urgent dental care hubs (UDHs) for active dental intervention. 
A reduction in non-essential patient attendance within secondary care dental settings and UDHs was encouraged as part of a nationwide effort to minimise transmission of COVID-19. Consequently, the AAA regimen provided a means of managing dental conditions for which urgent treatment was not indicated. Given the limited number of UDHs and initial uncertainty as to the timeframe and locations from which patients could seek urgent management of dental symptoms, the AAA regimen permitted remote prescription of analgesics and antibiotics for temporary relief of symptoms whilst such appointments were sought.

Despite the rationale underpinning the use of the AAA regimen, the challenges of performing patient assessment via telephone triage without clinical assessment provided the potential for inadvertent and/or inappropriate prescription of systemic antibiotics.

At the time of writing, England will imminently enter a second national lockdown. Furthermore, all other devolved nations of the UK have also implemented regional tiered restrictions aimed at reducing the transmission of the COVID-19 virus. It may therefore be postulated that further difficulty in accessing primary care dental settings may be experienced by patients in the near future.

This retrospective analysis sought to ascertain the effect of the AAA regimen upon the appropriateness of antibiotic prescribing for those patients attending for emergency dental extractions at the Department of Oral Surgery, King's College Dental Hospital (KCDH), London.

This has subsequently been used as a foundation upon which to discuss the potential factors that are likely to have had an effect upon the prescribing patterns of GDPs at this time and the possible future implications should the UK experience a secondary mandatory closure of primary care dental settings.

\section{Materials and methods}

Retrospective data collection for patients attending for emergency dental extractions was performed at the Department of Oral Surgery, KCDH. Data were collected by an oral surgery speciality registrar and a dental core trainee between March-June 2020 during KCDH's designation as a UDH.

The following data were collected:

- Gender

- Age

- American Society of Anaesthesiologists (ASA) physical status
- Diagnosis

- Whether AAA had been prescribed to patients before their attendance at $\mathrm{KCDH}$ for emergency dental extraction.

Subsequent to this, data analysis was performed to ascertain the proportion of patients who were prescribed antibiotics for each diagnosis provided.

All patient data were anonymised within an encrypted Microsoft Excel database held within a secured facility. Data analysis was performed by all members of the authorship.

\section{Results}

In total, 1,414 patients attended for emergency dental extraction. This group was comprised of 723 (51.1\%) male patients and 691 (48.9\%) female patients. The average age of the group was 45 years and 70 days. Overall, 907 (64\%) cases were treated by oral surgery speciality doctors, 293 (20.7\%) by oral surgery speciality registrars, $206(14.5 \%)$ by oral surgery consultants and the remaining $9(0.6 \%)$ by dental core trainees. Out of all emergency dental extractions performed, 1,241 (87\%) were non-surgical. Each patient was treated within 12 hours of telephone triage. All patients underwent treatment between March-June 2020.

The number of patients classified as ASA I and ASA II were 649 (45.8\%) and 639 (45.1\%) respectively. One hundred and twenty-four (8.7\%) patients were classified as ASA III.

One hundred and ninety-five (13.7\%) patients were classified as vulnerable patients according to PHE criteria. ${ }^{12} \operatorname{Six}(0.4 \%)$ patients presented with symptoms suggestive of COVID-19 positivity.

The most common diagnosis was chronic apical periodontitis, which was provided in 434 (33.7\%) of cases. In total, 348 (27.1\%) patients were diagnosed with acute apical periodontitis, 177 (13.7\%) with irreversible pulpitis and 125 (9.7\%) with an acute exacerbation of apical periodontitis. Other common diagnoses included vertical root fracture which was observed in 92 (7.2\%) cases, acute apical abscess diagnosed in 68 (5.3\%) cases and periodontal involvement in $49(3.8 \%)$ cases. Sixty-three (4.4\%) cases attended for management of post-operative symptoms following emergency dental extraction at $\mathrm{KCDH}$.

Overall, 471 (33.3\%) patients sought advice from their GDP before contacting KCDH's emergency dental triage service.
Prior to attending $\mathrm{KCDH}$ for emergency dental extraction, 1,195 (84.5\%) patients were prescribed or self-prescribed analgesia and $665(47 \%)$ of all patients were prescribed antibiotics by a GDP or GP before attending for emergency dental extractions. All patients included in this cohort were triaged via telephone by qualified dentists from members of all dental specialties based at $\mathrm{KCDH}$. All patients were subsequently assessed, diagnosed and treatment-planned upon the acute dental care department at $\mathrm{KCDH}$ before referral to the Department of Oral Surgery, KCDH for emergency dental extractions.

The proportion of patients who were prescribed systemic antibiotics for each dental condition is summarised in Table 1.

\section{Discussion}

Antimicrobial stewardship in dentistry

AMR remains a global public health challenge. Failure to address the challenge presented by AMR has been estimated to potentially result in ten million deaths every year globally by $2050,{ }^{13}$ whilst also contributing to the emergence of a number of antibiotic-resistant bacterial strains. ${ }^{3,45}$

The minimum inhibitory concentration (MIC) of an antibiotic is the lowest concentration at which an antibiotic can inhibit the growth of bacteria. ${ }^{14}$ AMR is assessed on the reduction of efficacy of an antibiotic against a pathogenic strain at MIC. ${ }^{15}$ Antimicrobials disrupt bacterial and fungal proliferation via a number of mechanisms including inhibition of bacterial wall synthesis, disruption of bacterial folic acid synthesis, inhibition of RNA transcription and prevention of subsequent DNA translation. AMR may occur through bacterial acquisition of exogenous resistance genes, ${ }^{16}$ chromosomal mutations ${ }^{17,18}$ or a combination of both mechanisms. ${ }^{19}$

Recent evidence demonstrates the potential of non-steroidal anti-inflammatory medications including ibuprofen and diclofenac to facilitate bacterial uptake of exogenous antibiotic resistance genes. ${ }^{20}$ Given that ibuprofen has been demonstrated as the preferred analgesic in managing dental pain ${ }^{21}$ and is readily available over the counter, antibiotic prescription for dental symptoms requires increasingly greater consideration in order to reduce the contribution of antibiotic prescribing in dentistry to AMR.

Between 2010-2017, dental prescription of antibiotics accounted for $10.8 \%$ of all antibiotic prescribing in England. During 
this time period, $64.8 \%$ of all oral antibiotics prescriptions by dentists were for amoxicillin, while metronidazole and erythromycin prescriptions accounted for $28 \%$ and $4.4 \%$, respectively, of all oral antibiotic prescriptions during the same time period. Overall, oral antibiotic prescribing by dentists reduced by $24.4 \%$ over the time period in contrast with a fall of $14.8 \%$ throughout all primary care settings in England during this time. ${ }^{1}$ Despite the decline in antibiotic prescribing in primary care dentistry, data persist suggesting antibiotics continue to be inappropriately prescribed in dentistry. ${ }^{2,22,23,24}$ Recent data have demonstrated that antibiotic prescribing in dentistry was disproportionately greater during the peak of the first wave of the pandemic in comparison to the preceding 28 months. ${ }^{25}$ Several factors are thought to contribute to prescribing behaviour, including the prescribing dentist's capabilities, and motivations and the influence of patients and peers. ${ }^{26}$

Antibiotic prescribing guidance for dentists in the UK is outlined by guidance published by the $\mathrm{FGDP}^{6}$, the $\mathrm{SDCEP}^{7}$ and the $\mathrm{NICE}^{8}$. Broadly, systemic antibiotic prescription in dentistry is recommended only in cases where systemic signs and symptoms of infection are observed.

\section{Challenges in successful implementation} of the AAA regimen

During the COVID-19 pandemic, the AAA regimen formed part of NHS England's COVID-19 guidance and standard operating procedure for remote patient management. ${ }^{11}$ This approach was proposed in order to reduce non-urgent and non-essential patient attendance within secondary dental care settings, thereby minimising COVID19 transmission. Whilst resources and appointment availability may continue to be limited, the AAA approach should not be considered as the primary approach for dental patient management. Rather, NHS England emphasise the need to 'weigh up the benefits of dental treatment against [COVID-19] transmission risk. The AAA approach, where indicated, should reflect FGDP, General Dental Council and SDCEP guidance.

Recent evidence regarding teledentistry demonstrates high levels of patient satisfaction $^{24}$ and early evidence suggests favourable opinions among dentists and dental professionals regarding its use. ${ }^{27,28,29}$ However, its value is limited primarily by infrastructural and organisational capabilities ${ }^{30}$ and patient access to the necessary technology. In the initial stages of the COVID-19 pandemic, teledentistry in primary and secondary dental

\begin{tabular}{|c|c|c|}
\hline Diagnosis & $\begin{array}{l}\text { Number of patients } \\
\text { (\% of all diagnoses) }\end{array}$ & $\begin{array}{l}\text { Number of antibiotic } \\
\text { prescriptions } \\
\text { (\% of cases prescribed } \\
\text { antibiotics for each diagnosis) }\end{array}$ \\
\hline Chronic apical periodontitis & $434(33.7 \%)$ & $210(48.4 \%)$ \\
\hline Acute apical periodontitis & $348(27.1 \%)$ & $180(51.7 \%)$ \\
\hline Irreversible pulpitis & $177(13.7 \%)$ & $81(45.7 \%)$ \\
\hline $\begin{array}{l}\text { Acute exacerbation of chronic apical } \\
\text { periodontitis }\end{array}$ & $125(9.7 \%)$ & $65(52 \%)$ \\
\hline Vertical root fracture & $92(7.1 \%)$ & $32(34.8 \%)$ \\
\hline Unrestorable caries & $91(7.1 \%)$ & $39(42.8 \%)$ \\
\hline Perio-endo lesion & $58(4.5 \%)$ & $30(51.7 \%)$ \\
\hline Periodontal involvement & $49(3.8 \%)$ & $12(24.5 \%)$ \\
\hline Cracked cusp syndrome & $31(2.4 \%)$ & $16(51.6 \%)$ \\
\hline Unrestorable retained roots & $28(2.2 \%)$ & $11(39.3 \%)$ \\
\hline Recurrent pericoronitis & $23(1.8 \%)$ & $19(82.6 \%)$ \\
\hline Unrestorable fracture & $15(1.1 \%)$ & $3(20 \%)$ \\
\hline Failed endodontic treatment & $13(1 \%)$ & $8(61.5 \%)$ \\
\hline Normal socket healing & $12(0.9 \%)$ & $1(8.3 \%)$ \\
\hline Periodontal abscess & $10(0.7 \%)$ & $3(30 \%)$ \\
\hline Failed dental extraction & $7(0.5 \%)$ & $4(57.1 \%)$ \\
\hline Chronic apical abscess & $6(0.4 \%)$ & $2(33.3 \%)$ \\
\hline $\begin{array}{l}\text { Post-operative infection following } \\
\text { dental extraction }\end{array}$ & $6(0.4 \%)$ & $0(0 \%)$ \\
\hline Trauma & $5(0.3 \%)$ & $3(60 \%)$ \\
\hline Bony sequestration & $3(0.2 \%)$ & $0(0 \%)$ \\
\hline Acute pericoronitis & $2(0.15 \%)$ & $2(100 \%)$ \\
\hline Radicular cyst & $2(0.15 \%)$ & $1(50 \%)$ \\
\hline Oro-antral communication & $2(0.15 \%)$ & $0(0 \%)$ \\
\hline Gingival overgrowth & $1(0.15 \%)$ & $1(100 \%)$ \\
\hline Disto-cervical caries & $1(0.15 \%)$ & $0(0 \%)$ \\
\hline Intra-cortical infection & $1(0.07 \%)$ & $0(0 \%)$ \\
\hline Residual post-operative swelling & $1(0.07 \%)$ & $0(0 \%)$ \\
\hline Reversible pulpitis & $1(0.07 \%)$ & $0(0 \%)$ \\
\hline External cervical resorption & $1(0.07 \%)$ & $0(0 \%)$ \\
\hline Over-erupted tooth & $1(0.07 \%)$ & $0(0 \%)$ \\
\hline Ulceration of oral mucosa & $1(0.07 \%)$ & $0(0 \%)$ \\
\hline Gingival epulis & $1(0.07 \%)$ & $0(0 \%)$ \\
\hline Lichen planus & $1(0.07 \%)$ & $0(0 \%)$ \\
\hline Granulation tissue & $1(0.07 \%)$ & $0(0 \%)$ \\
\hline
\end{tabular}

care settings was largely limited to telephone

Patients may provide an inaccurate history or withhold information regarding consultations. 
symptoms..$^{31,32}$ This combined with the inability of a clinician to perform clinical examination during telephone triage creates potential for inadvertent misdiagnosis. When in doubt, NHS England recommend 'shared decisionmaking to determine whether care should continue remotely or face-to-face. ${ }^{11}$

The population of inner-city and Greater London totals approximately 8.9 million. In total, this population was initially served by seven UDHs. As such, during the mandated temporary closure of dental practices nationwide, access to emergency dental services was limited. This is likely to have been echoed in many parts of the country. The limited access to care and uncertainty regarding appointment availability at UDHs may have driven the prophylactic prescription of antibiotics by GDPs or GPs for such patients.

\section{Antibiotic prescription in exceptional circumstances}

This approach may be justified when dental symptoms are nonspecific or suggestive of localised or systemic infection. Consequently, this may account for the greater proportions of patients who were prescribed antibiotics before contacting $\mathrm{KCDH}$ for suspected pericoronitis, acute apical abscess, fascial space abscess and failed endodontic treatment. Given the limited number of emergency appointments in primary dental care settings during this time period, antibiotic prescription in such cases may be justified. Furthermore, evidence suggests that antibiotic prescription for symptomatic apical periodontitis may be effective $\mathrm{e}^{33,34}$ though further research is indicated. ${ }^{35}$

Antibiotics have been demonstrated as ineffective and inappropriate for the management of irreversible pulpitis. ${ }^{36,37}$ Pulpitic symptoms are often easily diagnosed. Some clinical justification for antibiotic prescription may tenuously be provided for the management of suspected infection in exceptional circumstances, despite contravening well-established antibiotic stewardship guidance. These data demonstrate that $51.7 \%$ of patients diagnosed with irreversible pulpitis were prescribed systemic antibiotics. Ordinarily, this figure could be considered disproportionate, but may reflect uncertainties regarding resumption of normal care or access to emergency appointments.

In total, $51.6 \%$ of patients diagnosed with cracked cusp syndrome received antibiotic prescription, which as with pulpitis, is ineffective in its management. Cracked cusp syndrome is often exacerbated when biting on the affected tooth and may be inadvertently mistaken for apical periodontitis. This may explain the inappropriate prescription of antibiotics in a limited number of exceptional circumstances. However, given that cracked cusp syndrome is often poorly localised and mimics pulpitic pain ${ }^{38}$ justification cannot be provided for the disproportionate numbers of patients that were prescribed antibiotics.

Overall, $57.1 \%$ of patients who experienced failed extraction were subsequently prescribed antibiotics. This cohort of patients were likely to have suffered a prolonged period of pain that may have justified the extraction or symptoms that may have been exacerbated by the failed extraction. Antibiotic prescription following failed extraction alone would not have alleviated such symptoms. There may be some justification for the prescription of antibiotics in light of pre-existing medical conditions. In this cohort, five patients were classified ASA II and the remaining two patients ASA I. As such, this lends less justification for antibiotic prescription in this cohort of patients.

Positive findings have emerged from this review. Rates of antibiotic prescription remained low among patients diagnosed with conditions in which antibiotic prescription would have had provided no symptomatic relief. No patients diagnosed with localised post-extraction infection were prescribed antibiotics, whilst only one patient diagnosed with normal socket healing was prescribed antibiotics. These figures should be approached with some caution given the smaller sample sizes.

It is unclear as to when widespread knowledge regarding UDHs was disseminated to and among GDPs. This, coupled with the aforementioned challenges of remote diagnosis, is likely to have influenced antibiotic prescribing at this time.

Given the increased risk of transmission of COVID-19 within secondary care, many patients may have declined face-to-face assessment and instead requested antibiotics or placed undue pressure upon dental professionals to provide antibiotic prescription. Given the exceptional circumstances, attending dentists may have had a lower threshold for antibiotic prescription, which may not have necessarily been reciprocated outside of the COVID-19 pandemic.

Prior to implementing the modified treatment pathway ${ }^{39}$ for patients attending for emergency dental extraction, the telephone number to access the Acute Dental Care service was already publicly available via the hospital trust website. During the time period for which these data were collected, attending dentists, oral surgeons and dental nurses were anecdotally informed by a number of patients that this telephone number was circulated via social media and by GDPs at the height of the first wave of the pandemic. Awareness of this point of access may have lowered the threshold for antibiotic prescription by GDPs, given the knowledge that emergency dental care could be accessed with relative ease at a large UDH such as $\mathrm{KCDH}$.

\section{Conclusion}

Our findings suggest that the AAA regimen has inadvertently contributed to inappropriate prescription of systemic antibiotics by GDPs. However, this regimen formed part of an urgent response to managing acute dental conditions during an exceptional period. Given the imposed inability of GDPs to clinically examine patients at this time, it may be somewhat unfair to presume that these data are reflective of widespread antibiotic prescribing practices in primary dental care settings or that one single factor has contributed towards this. Additionally, this dataset is limited in that it considers patients attending for dental extraction rather than for restorative dental treatment. Several factors contributing towards inappropriate antibiotic prescription have been established. The aforementioned uncertainties regarding access to treatment are likely to have amplified the role of these factors. ${ }^{37}$

These findings are not necessarily suggestive of antibiotic prescribing practices in general dental practice. While all patients included in this analysis were prescribed antibiotics by GDPs, a number of patients may have been initially referred by dentists in secondary care undertaking the telephone triage service to seek antibiotic prescription in primary care where indicated.

Although dental practices have been permitted to resume normal activity from 4 June 2020, new standard operating procedure guidance has resulted in a significant reduction of outpatient activity. Greater consideration must be placed towards antibiotic prescription in cases where it is likely to be ineffective, despite extended waiting list times. The adoption of online video conferencing may also go some way to providing GDPs with a means of 
achieving more accurate diagnosis, though this may be limited by financial and infrastructural constraints for dental professionals and patients alike. At all times, dental practitioners should remain mindful of antibiotic stewardship guidance and the contribution of indiscriminate antibiotic prescription to global AMR.

\section{References}

1. Thornhill M H, Dayer M J, Durkin M J, Lockhart P B, Baddour L M. Oral antibiotic prescribing by NHS dentists in England 2010-2017. Br Dent J 2019; 227: 1044-1050.

2. Bansal R, Jain A, Goyal M, Singh T, Sood H, Malviya $H$ S. Antibiotic abuse during endodontic treatment: $A$ contributing factor to antibiotic resistance. J Family Med Prim Care 2019; 8: 3518-3524.

3. Pachori P, Gothawal R, Gandhi P. Emergence of antibiotic resistant Pseudomonas aeruginosa in intensive care unit: a critical review. Genes Dis 2019; 6 : 109-119.

4. Li Z, Cao Y, Yi L, Liu J-H, Yang Q. Emergent Polymyxin Resistance: End of an Era? Open Forum Infect Dis 2019; DOI: 10.1093/ofid/ofz368.

5. Niestepski S, Harnisz M, Korzeniewska E et al. The emergence of antimicrobial resistance in environmental strains of the Bacteroides fragilis group. Environ Int 2019; 124: 408-419

6. Faculty of General Dental Practitioners. Antimicrobial Prescribing for General Dental Practitioners. 2016. Available online at https://www.fgdp.org.uk/ antimicrobial-prescribing-standards/title-page (accessed December 2020).

7. Scottish Dental Clinical Effectiveness Programme. Drug Prescribing for Dentistry: Dental Clinical Guidance, Third Edition. 2016. Available at https://www.sdcep. org.uk/wp-content/uploads/2016/03/SDCEP-DrugPrescribing-for-Dentistry-3rd-edition.pdf (accessed December 2020).

8. National Institute for Health and Care Excellence. Antimicrobial Stewardship: Systems and Processes for Effective Antimicrobial Medicine Use. 2018. Available at https://www.nice.org.uk/guidance/ng15/ resources/antimicrobial-stewardship-systems-andprocesses-for-effective-antimicrobial-medicine-usepdf-1837273110469 (accessed December 2020).

9. Public Health England. Dental Antimicrobial Stewardship: Toolkit. 2020. Available online at https://www.gov.uk/guidance/dental-antimicrobialstewardship-toolkit (accessed December 2020).

10. Chief Dental Officer England. Preparedness letter for primary dental care (March 2020). 2020. Available online at https://www.england.nhs.uk/coronavirus/ publication/preparedness-letters-for-dental-care/ (accessed December 2020).

11. NHS England. COVID-19 Guidance and Standard Operating Procedure (Version 3). 2020. Available at https://www.england.nhs.uk/coronavirus/wp-content/ uploads/sites/52/2020/06/C0581-covid-19-urgentdental-care-sop-update-16-june-20-.pdf (accessed December 2020)

12. Public Health England. Guidance on Shielding and Protecting People who are Clinically Extremely Vulnerable from COVID-19. 2020. Available online at https://www.gov.uk/government/ publications/guidance-on-shielding-and-protectingextremely-vulnerable-persons-from-covid-19/ guidance-on-shielding-and-protecting-extremelyvulnerable-persons-from-covid-19 (accessed December 2020).

13. Review on Antimicrobial Resistance. Tackling Drug-Resistant Infections Globally: Final Report and Recommendations. 2016. Available at https://amrreview.org/sites/default/files/160518_Final\%20paper_ with\%20cover.pdf (accessed December 2020).

14. Marsh P, Martin M. Oral Microbiology. New York: Elsevier, 2009

15. Martínez $J \mathrm{~L}$, Baquero F. Emergence and spread of antibiotic resistance: setting a parameter space. Ups J Med Sci 2014; 223: 68-77.

16. Yousuf M, Iuliani I, Veetil R T, Seshasayee A S N, Sclavi B, Lagomarsino M C. Early fate of exogenous promoters in E. coli. Nucleic Acids Res 2020; 48: 2348-2356.

17. Davies J, Davies D. Origins and evolution of antibiotic resistance. Microbio/ Mol Biol Rev 2010; 74: 417-433.

18. Hughes D, Andersson D I. Evolutionary trajectories to antibiotic resistance. Annu Rev Microbiol 2017; 71: 579-596.

19. Porse A, Jahn L J Ellabaan M M H Sommer M O A Dominant resistance and negative epistasis can limit the co-selection of de novo resistance mutations and antibiotic resistance genes. Nat Commun 2020; 11 : 1199

20. Wang Y, Lu J, Engelstädter J et al. Non-antibiotic pharmaceuticals enhance the transmission of exogenous antibiotic resistance genes through bacterial transformation. ISME J 2020; 14: 2179-2196.

21. Moore P, Ziegler K, Lipman R D, Aminoshariae A, Carrasco-Labra A, Mariotti A. Benefits and harms associated with analgesic medications used in the management of acute dental pain. J Am Dent Assoc 2018; 149: 256-268.

22. Dailey $Y$, Martin M. Are antibiotics being used appropriately for emergency dental treatment? Br Dent J 2001; 191: 391-393

23. Cope A L, Chestnutt I G, Wood F, Francis N A. Dental consultations in UK general practice and antibiotic prescribing rates: a retrospective cohort study. $\mathrm{Br} J \mathrm{Gen}$ Pract 2016; DOI: 10.3399/bjgp16X684757.

24. Bird L, Landes D, Robson T, Sturrock A, Ling J. Higher antibiotic prescribing propensity of dentists in deprived areas and those with greater access to care in the North East and Cumbria, UK. Br Dent J 2018; 225: 517-524.

25. Wordley V, Shah S, Thompson W. Increased antibiotics use. Br Dent J 2020; 229: 266.

26. Hellyer $P$. Antibiotic resistance is a global health problem. Br Dent J 2020; 229: 473.

27. Rahman N, Nathwani S, Kandiah T. Teledentistry from a patient perspective during the coronavirus pandemic. $\mathrm{Br}$ Dent J 2020; D0I: 10.1038/s41415-020-1919-6.

28. van der Zande M M, Gorter R C, Bruers J J M, Aartman I H A, Wismeijer D. Dentists' opinions on using digital technologies in dental practice. Community Dent Oral Epidemiol 2017; 46: 143-153.

29. Weintraub J, Edwards L, Brame J et al. Teledentistry Knowledge and Attitudes: Perspectives on the role of dental hygienist. J Dent Hyg 2020; 94: 13-21.

30. Estai M, Kruger E, Tennant M, Bunt S, Kanagasingam Y. Challenges in the uptake of telemedicine in dentistry. Rural Remote Health 2016: 16: 168-172.

31. Peters S, Rogers A, Salmon P et al. What do patients choose to tell their doctors? Qualitative analysis of potential barriers to reattributing medically unexplained symptoms. J Gen Intern Med 2009; 24: 443-449.

32. Beverly E A, Ganda O P, Ritholz M D et al. Look who's (not) talking: diabetic patients' willingness to discuss self-care with physicians. Diabetes Care 2012; 35: $1466-1472$.

33. Skucaite N, Peciuliene V, Vitkauskiene A, Machiulskiene V. Susceptibility of Endodontic Pathogens to Antibiotics with Symptomatic Apical Periodontitis. J Endod 2010; 36: 1611-1616

34. Lee L, Lee $Y$, Hsiao S H, Lin H P. Bacteria in the apical root canals of teeth with apical periodontitis. J Formos Med Assoc 2017; 116: 448-456.

35. Veitz-Keenan A, De Bartolo A M. Insufficient evidence of the effect of systemic antibiotics on adults with symptomatic apical periodontitis or acute apical abscess. Evid Based Dent 2014; 15: 104-105.

36. Hoskin E, Veitz-Keenan A. Antibiotics are not useful to reduce pain associated with irreversible pulpitis. Evid Based Dent 2016; 17: 81-82.

37. Agnihotry A, Thompson W, Fedorowicz Z, van Zuuren E J, Sprakel J. Antibiotic use for irreversible pulpitis. Cochrane Database Syst Rev 2019; DOI: 10.1002/14651858.CD004969.pub5.

38. Carotte P. Endodontics: Part 3 Treatment of endodontic emergencies. Br Dent J 2004; 197: 299-305.

39. Shah A, Bryant C, Patel J et al. COVID-19: establishing an oral surgery led urgent dental care hub. Br Dent $J 2020$; 228: 957-963. 